\title{
Forecasting U.S. Recessions with a Large Set of Predictors
}

\author{
Paolo Fornaro \\ University of Helsinki and HECER
}

Discussion Paper No. 389

March 2015

ISSN 1795-0562

HECER - Helsinki Center of Economic Research, P.O. Box 17 (Arkadiankatu 7), FI-00014 University of Helsinki, FINLAND, Tel +358-2941-28780, E-mail info-hecer@helsinki.fi, Internet www.hecer.fi 


\title{
Forecasting U.S. Recessions with a Large Set of Predictors*
}

\begin{abstract}
In this paper, I use a large set of macroeconomic and financial predictors to forecast U.S. recession periods. I adopt Bayesian methodology with shrinkage in the parameters of the probit model for the binary time series tracking the state of the economy. The in-sample and out-of-sample results show that utilizing a large cross-section of indicators yields superior U.S. recession forecasts in comparison to a number of parsimonious benchmark models. Moreover, data rich models with shrinkage manage to beat the forecasts obtained with the factor-augmented probit model employed in past research.
\end{abstract}

JEL Classification: C11, C25, E32, E37

Keywords: Bayesian shrinkage, business cycles, probit model, large cross-sections

Paolo Fornaro

Department of Political and Economic Studies

University of Helsinki

P.O. Box 17 (Arkadiankatu 7)

FI-00014 University of Helsinki

FINLAND

e-mail: paolo.fornaro@helsinki.fi

* I wish to thank my supervisors Henri Nyberg and Antti Ripatti for their invaluable comments and suggestions. Moreover, I would like to thank Jani Luoto and the other participants at University of Helsinki seminars for their constructive comments. Financial support from the Yrjö Jahnsson foundation, the OP-Pohjola Group Research foundation, the Finnish Ministry of Education and Culture and the Academy of Finland is gratefully acknowledged. 


\section{Introduction}

Recession forecasting is a key activity performed by numerous economic institutions. Knowing whether in the next month or next year the economy will be in an expansion or recession is an important piece of information for policymakers, investors and households. For example, government authorities can tailor their spending with the knowledge of how soon the economy will return to expansion, while central banks can review their monetary policy in the light of future expected business cycle conditions.

In the applied econometric literature, recession forecasting has typically been based on binary response frameworks, such as probit or logit models. In these studies, only a few predictive variables at a time are used to forecast recession periods. It has generally been found (see, e.g, Dueker (1997), and Estrella and Mishkin (1998)) that the spread between the ten-year Treasury bond rate and the three-month Treasury bill rate is the best leading indicator of the U.S. recessions. Furthermore, Wright (2006) finds that the level of the federal funds rate has some additional predictive power over and above the term spread, whereas similar results have been found for the stock market returns in Estrella and Mishkin (1998) and Nyberg (2010).

In this paper, I propose a novel approach based on Bayesian shrinkage allowing for the presence of a large number of predictors in the probit model. Using a high-dimensional monthly dataset, I compute 1, 6, 9 and 12-month-ahead recession forecasts from a set of models which differ in the number of explanatory variables used. The parsimonious benchmark models include the variables that have been found useful recession leading indicators, such as the term spread.

Despite the growing interest in predicting recessions, the use of large datasets for this purpose has not been widespread. Nevertheless, there have been a few notable examples, such as Chen, Iqbal, and Lai (2011), where the authors include estimated latent factors extracted from a large dataset in the probit model. Fossati (2013) also proposes the use of the constructed macroeconomic factors as predictors, even though he focuses on smaller datasets than Chen et al. (2011) when estimating the dynamic factors. Recently, Christiansen, Eriksen, and Møller (2014) use common factors in the probit models to test the usefulness of sentiment variables. In contrast to these above-mentioned binary response models, the predictive frameworks for continuous real-valued dependent variables, such as GDP growth, containing a large number of predictors have been commonly used in the previous literature since the seminal paper by Stock and Watson 
(2002). They introduced the use of principal components, estimated from a large macroeconomic dataset, to forecast variables of interest (such as industrial production or inflation). Dynamic factor settings have not been the only class of models used in macroeconomic forecasting with large datasets. For example, De Mol, Giannone, and Reichlin (2008) propose Bayesian shrinkage as an alternative to principal components, while Banbura, Giannone, and Reichlin (2010) forecast macroeconomic variables using a large Bayesian vector autoregression.

I apply a methodology similar to the one presented in De Mol et al. (2008) to shrink the parameters of the explanatory variables toward zero, leading to a ridge regression-type setting. The probit model is estimated with Bayesian methodology via data augmentation as in Albert and Chib (1993). The main contribution to the previous literature is that I am able to estimate a probit model with a large number of predictors via Bayesian shrinkage. This is a key distinction from other works concerning forecasting recession periods using factor-based models, where the information contained in large datasets is condensed in a few unobservable common factors. My approach has the desirable property of allowing to assess the effect of individual variables, with convenient interpretation of the parameter estimates. Another problematic feature of factor models is that they require a two-step estimation procedure (with potential issues related to the generated regressor problem) but also produce predictors which have no clear economic interpretation. Furthermore, another contribution on the research of binary response models is the use of informative priors. This is different from what is done in, e.g., Albert and Chib (1993) and Chauvet and Potter (2005), where the authors rely on flat priors. In my case, I use a shrinkage prior, i.e. I center the prior distribution of the parameters at zero, with the variance of the prior distribution used to control how much the parameters are shrunk.

In my empirical application to U.S. recession periods, I find that the probit models containing a large set of predictors outperform the more parsimonious models. This result, however, holds only in the case where we shrink the parameters of the model toward zero. The overall superior forecasting performance is not only reflected in statistical criteria, but models incorporating a large set of explanatory variables give us predictions that are informative for decision making. Moreover, the large forecasting models manage to beat factor-based recession forecasts, providing a competitive alternative for the use of large datasets in recession prediction. 
The remainder of the paper is structured as follows: In Section 2, I introduce the model and the shrinkage methodology. In Sections 3 and 4, I briefly describe the dataset and report the empirical results. Section 5 concludes.

\section{Methodology}

\subsection{Probit Model}

Following the modeling approach by Albert and Chib (1993), I consider probit models estimated with Bayesian methodology. In particular, I use the data augmentation technique to obtain posterior draws for the model parameters and the latent variable underlying the binary recession indicator.

Throughout this study, I am interested in forecasting a binary variable, $y_{t}, t=$ $1,2, \ldots, T$, which can take the value one or zero. In our U.S. recession forecasting application, following the usual practice in macroeconomic research, $y_{t}$ is thus the NBER recession indicator defined as

$$
y_{t}=\left\{\begin{array}{l}
1, \text { if the U.S. economy is in a recession at time } t \\
0, \text { if the U.S. economy is in an expansion at time } t .
\end{array}\right.
$$

Furthermore, I assume that the realized values of $y_{t}$ are based on a latent variable $z_{t}$ defining the values of (2.1) as follows:

$$
y_{t}=\left\{\begin{array}{l}
1, \text { if } z_{t}>0 \\
0, \text { if } z_{t} \leq 0
\end{array}\right.
$$

In other words, negative values of $z_{t}$ imply $y_{t}=0$ (i.e. expansions), and vice versa for recessions.

In the probit model, I use $p$ lags of the explanatory variables to forecast recessions, so our model for the latent variable $z_{t}$ becomes:

$$
z_{t}=X_{t}^{\prime} \beta+u_{t}
$$

where $X_{t}=\left(1, x_{t-1}^{\prime}, \ldots, x_{t-p}^{\prime}\right)^{\prime}$ is $(n p+1) \times 1$ vector and $u_{t}$ is the error term which follows a standard normal distribution. Due to the form of (2.3), $\beta$ contains the constant together with the coefficients associated with the predictors and their lags. Model (2.3) 
can be rewritten using a matrix notation as:

$$
Z=X \beta+U
$$

where the vector $Z=\left(z_{1}, \ldots, z_{T}\right)^{\prime}$ is $(T \times 1)$ vector, $X=\left(X_{1}, \ldots, X_{T}\right)^{\prime}$ is $(T \times n p+1)$ matrix and $U=\left(u_{1}, \ldots, u_{T}\right)^{\prime}$ is a $(T \times 1)$ vector.

From (2.2) and (2.3), we obtain:

$$
E_{t-1}\left(y_{t}\right)=P\left(z_{t} \geq 0 \mid X_{t}, \beta\right)=\Phi\left(X_{t}^{\prime} \beta\right)
$$

where $\Phi(\cdot)$ is the cumulative standard normal distribution function leading to the probit model. Notice that following the properties of the Bernoulli distribution, the conditional expectation $E_{t-1}\left(y_{t}\right)$, i.e. the expected value of the recession indicator conditional on the information set at time $t-1$, is equal to the conditional probability $P\left(z_{t} \geq 0 \mid X_{t}, \beta\right)$. The estimation of model (2.4) is carried out by Gibbs sampling. The details of the sampler are given in Section 2.3.

\subsection{Shrinkage Estimator}

Similarly as Albert and Chib (1993), I assume that in (2.4) the error term $U$ is multinormally distributed with mean 0 and identity variance-covariance matrix $\mathrm{I}_{T}$ (i.e. $\left.U \sim N\left(0, \mathrm{I}_{T}\right)\right)$. To derive the conditional posteriors for $\beta$ and $Z$, I follow the presentation of Zellner (1971).

Instead of using a flat non-informative prior for $\beta$ (as is often done in the literature), I impose the following prior

$$
p(\beta) \propto|A|^{1 / 2} \exp \left[-\frac{1}{2}(\beta-\bar{\beta})^{\prime} A(\beta-\bar{\beta})\right]
$$

where $A$ is a nonsingular matrix (in our case it is set to $\frac{1}{\lambda} \mathrm{I}_{K}$, with $K=n p+1$ i.e. the number of parameters). This implies that the prior for $\beta$ can be written compactly as $\beta \sim N\left(\bar{\beta}, A^{-1}\right)$. The likelihood for the latent variable $Z$, conditional on $\beta$, is given by

$$
p(Z \mid X, \beta) \propto \exp \left[-\frac{1}{2}(Z-X \beta)^{\prime}(Z-X \beta)\right]
$$

We combine the likelihood with the prior to get

$$
p(\beta \mid X, Z) \propto \exp \left\{-\frac{1}{2}\left[(Z-X \beta)^{\prime}(Z-X \beta)+(\beta-\bar{\beta})^{\prime} A(\beta-\bar{\beta})\right]\right\} .
$$


Notice that

$$
\begin{array}{r}
(\beta-\bar{\beta})^{\prime} A(\beta-\bar{\beta})+(Z-X \beta)^{\prime}(Z-X \beta)= \\
\beta^{\prime}\left(A+X^{\prime} X\right) \beta-2 \beta^{\prime}\left(A \bar{\beta}+X^{\prime} Z\right)+Z^{\prime} Z+\bar{\beta} A \bar{\beta}= \\
(\beta-\tilde{\beta})^{\prime}\left(A+X^{\prime} X\right)(\beta-\tilde{\beta})+Z^{\prime} Z+\bar{\beta}^{\prime} A \bar{\beta}-\tilde{\beta}^{\prime}\left(A+X^{\prime} X\right) \tilde{\beta},
\end{array}
$$

where $\tilde{\beta}=\left(A+X^{\prime} X\right)^{-1}\left(A \bar{\beta}+X^{\prime} Z\right)$, allowing us to rewrite the conditional posterior of $\beta$ as

$$
p(\beta \mid X, Z) \propto \exp \left\{-\frac{1}{2}\left[n^{\prime} c+(\beta-\tilde{\beta})^{\prime}\left(X^{\prime} X+A\right)(\beta-\tilde{\beta})\right]\right\},
$$

where $n^{\prime} c=Z^{\prime} Z+\bar{\beta}^{\prime} A \bar{\beta}-\tilde{\beta}^{\prime}\left(A+X^{\prime} X\right) \tilde{\beta}$ does not contain $\beta$ and we can drop it from the previous equation.

By looking at the right-hand side of (2.6), we see that the posterior of $\beta$, conditional on the latent variable $Z$, follows a multivariate normal with mean $\tilde{\beta}$ and variance $\left(A+X^{\prime} X\right)^{-1}$. Notice that setting $A=\frac{1}{\lambda} \mathrm{I}_{K}$ and $\bar{\beta}=0$ (i.e. I impose shrinkage on the parameters), we get that $\tilde{\beta}=\left(X^{\prime} X+\frac{1}{\lambda} \mathrm{I}_{K}\right)^{-1}\left(X^{\prime} Z\right)$, which is the same estimate obtained by a penalized ridge regression in a frequentist setting as pointed out in De Mol et al. (2008). In particular, $\tilde{\beta}=\beta_{\text {Ridge }}$ is the parameter estimate that minimizes the standard sum of squared errors plus the penalization term $1 / \lambda \sum_{j}^{n p} \beta_{j}^{2}$. The value of $\lambda$ determines how much we are shrinking the parameters: with a large $\lambda$ we are imposing a looser shrinkage, giving us estimates that are very close to the OLS solution, while a low value of $\lambda$ will lead to coefficients being very close to 0 . This is reflected in the minimization problem, where a very large value of $\lambda$ will lead the penalization term to be zero, and hence the estimator reduces to the standard OLS formula.

To set the hyperparameter $\lambda$, I follow a similar approach as in De Mol et al. (2008). I first compute the in-sample fit of the model with a few explanatory variables, and set $\lambda$ for richer models in a way to achieve equal in-sample fit. It is expected that $\lambda$ should decrease with model size, indicating a need of a tighter shrinkage for models with a large number of predictors. To account for the fact that higher order lags of the predictors should have a lower forecasting power, I modify the priors in such a way to impose tighter shrinkage on lags further in the past. To achieve this, I set $A=\frac{1}{\lambda} \mathrm{J}_{K}$, where the matrix $\mathrm{J}_{K}$ is diagonal with ones for the elements corresponding to the first lag of the variables, and higher values on the diagonal elements corresponding to the subsequent lags. A common choice is to set the diagonal elements of $\mathrm{J}_{K}$ as $p^{2}$, where $p$ 
indicates the lag length of the predictors.

\subsection{Estimation of the Probit Model}

The probit model (2.4) can be estimated using the Gibbs sampler suggested by Albert and Chib (1993), which takes the following form. Given the initial values of $z_{t}$ and $\beta$, in steps $j=1, \ldots, m$ :

1. Draw $z_{t}^{j}$, conditional on $\beta^{j-1}$, from a truncated normal with mean $X_{t}^{\prime} \beta^{j-1}$ and standard deviation 1 , on the interval $(-\infty, 0)$ if $y_{t} \leq 0$, otherwise draw $z_{t}^{j}$ from a truncated normal on the interval $(0, \infty)$

2. Draw $\beta^{j}$, conditional on $z_{t}^{j}$, from a multivariate normal with mean $\tilde{\beta}^{j}$ and variance $\left(\frac{1}{\lambda}+X^{\prime} X\right)^{-1}$. The form of the conditional posteriors are presented in Section 2.2. I repeat the above iterations $m$ times. In this application, $m$ is set to 10000 with an initial burn-in period of 1000 iterations, giving us a total of 9000 draws.

\subsection{Forecast Computation}

The computation of recession forecasts using model (2.4) is fairly straightforward, provided the estimated parameters. Once I have carried out the estimation with the Gibbs sampler, I have $m_{e f}=m-1000=9000$ valid draws for $\beta$ and $Z$. Based on those, I obtain $m_{e f}$ forecasts for the latent variable $z_{t}$. One-month-ahead forecast is obtained in the following way. First, compute

$$
\widehat{z}_{T_{\text {in }}+1}^{j}=X_{T_{i n}}^{\prime} \beta^{j}
$$

where $T_{i n}$ is our last in-sample observation. From these $m_{e f}$ forecasts of the latent variable, we obtain $m_{e f}$ probabilities of recession, denoted as $\widehat{P}_{T_{i n}+1}$,

$$
\widehat{P}_{T_{i n}+1}^{j}=\Phi\left(X_{T_{i n}}^{\prime} \widehat{\beta^{j}}\right)
$$

where $j=1, \ldots, m_{e f}$. I follow Dueker (1997) and Chauvet and Potter (2005) and obtain one-month-ahead point forecasts by averaging the predictions given by (2.8) over $m_{e f}$. That is,

$$
\widehat{P}_{T_{i n}+1}=\frac{1}{m_{e f}} \sum_{j=1}^{m_{e f}} \widehat{P}_{T_{i n}+1}^{j} .
$$


Multistep-ahead forecasts can be computed in a direct fashion (cf. the discussion of direct and iterative multistep forecasting methods in the usual AR model, e.g., in Marcellino, Stock, and Watson (2006)). This means that, for $h$-months-ahead forecasts, I estimate a model similar as (2.3):

$$
z_{t}=X_{t-h}^{\prime} \beta+u_{t}
$$

where $X_{t-h}^{p}=\left(1, x_{t-h}^{\prime}, x_{t-h-1}^{\prime}, \ldots, x_{t-h-p}^{\prime}\right)^{\prime}$. This procedure gives horizon-specific parameters estimates, from which I can compute the forecasts by

$$
\widehat{P}_{T_{i n}+h}^{j}=\Phi\left(X_{T_{i n}-h}^{\prime} \widehat{\beta}^{j}\right)
$$

Finally, the point forecasts $\widehat{P}_{T_{i n}+h}$ are obtained by averaging over the number of draws in a similar way as in (2.9).

\section{Data}

I compute recession forecasts using a monthly U.S. data. My dataset starts in February 1959 and ends in February 2009. The predictive variables are taken from Stock and Watson (2012) dataset, which includes 106 variables, ranging from real activity indicators, price indices and financial variables.

I use seven probit models, all with $p=3$ lags to account for the information of the previous quarter (three-month period). Variables are transformed to achieve stationarity and standardized to have mean 0 and standard deviation 1 (this data transformation is required for the factor extraction). All models including different predictors are subsets of the Stock and Watson data. The models are:

- Model (SP) contains the predictors considered the best leading indicators in recession forecasting, i.e. the spread between between long-term and short-term interest rates, and the federal funds rate, (see, e.g., Wright (2006)).

- A small model (SMALL), containing 5 variables including the spread between 10-year government bond and 3-month Treasury bill rates, the effective federal funds rate, industrial production, non-farm employment and the consumer price inflation (all items).

- A model (MEDIUM) containing 10 variables. This set of predictors includes the variables of SMALL plus M2 money aggregate, total reserves, real consumption, 
capacity utilization and the effective exchange rate.

- A model (LARGE) which comprises 20 variables. In addition to the variables of MEDIUM, I add average hourly earnings, M1 money aggregate, Standard \& Poor stock market returns, Yields on 5 years US Treasury Bond, the National Association of Purchasing Managers and the producer price indices, housing starts, help wanted and civilian labor force indices and consumer credit outstanding.

- A model with 30 variables (VLARGE), which adds to the previous datasets the AAA bonds yields, BAA bond yields, the Bureau of Labor Statistics spot market price index, oil price, the dollar pound exchange rate, the Dow Jones stock market returns, the consumer expectation index, new orders, commercial and industrial loans and unemployment duration.

- A very large model (GIANT) which contains all 106 variables of the Stock and Watson(2012) macroeconomic dataset. This includes all the predictive variables listed above.

Finally, it is of interest to compare the forecasting performance of our models against the factor-augmented probit models by Chen et al. (2011) and Christiansen et al. (2014). They provide a natural comparison, given that factor models are commonly used to incorporate large datasets' information in macroeconomic analysis. In practice, following their methodology, I use a two-step procedure where in the first step a set of common factors is extracted using the principal component-based estimator presented in Stock and Watson (2002), and in the second step, I employ the estimated factors as predictors in the usual probit model. The factors are extracted from the whole dataset containing 106 variables, examined in model GIANT, and the number of factors is selected using the information criterion proposed in Bai and $\mathrm{Ng}$ (2002). I find that the optimal number of factors is 4 , giving us a parsimonious model and hence I do not apply shrinkage to it. I denote this model as FACTORS hereafter.

It is also worth noting that in recent years, there has been a surge in the use of dynamic probit models to forecast recession periods. That is, the lagged values of the recession indicator $y_{t}$ are used as predictors in the probit model. Notable examples are Kauppi and Saikkonen (2008), Startz (2008), Chauvet and Potter (2005) and Nyberg (2010, 2014). In this study, I follow another approach where the use of a large set of predictors is seen as an alternative to the dynamic models. In particular, similarly as including the lags of $y_{t}$, I am taking the coincident state of the economy into account 
at the time I make the prediction by adding coincident economic indicators (and their lags), like industrial production and retail sales, to our predictive information set. These coincident variables are highly correlated with the recession indicator, as the latter is based on their values, and hence, in principle, including the past values of the recession indicator would not increase the predictive power significantly. As discussed in Chauvet and Potter (2005), the Bayesian estimation of dynamic probit models, even for rather simple dynamics, is computationally burdensome, making this kind of models undesirable when we are interested in a large number of predictors. Finally, the values of the binary recession indicator are available after months' delay. Thus, including coincident variables directly in the probit model appears to be an interesting alternative to dynamic probit models.

\section{Empirical Results}

\subsection{Forecast Evaluation}

As described in Section 3, the sample period ranges from February 1959 to February 2009. The in-sample period is set to end in November 1979 (250 observations), while the remaining observations are used to evaluate out-of-sample forecasts. In this way, I obtain more than half of the sample for forecast evaluation. This time span includes five recessions: two recessions in the early 1980s, one in the early 1990s, the short recession of the beginning of 2000s and finally the recent economic crisis which started in December 2007. I compute forecasts using an expanding window approach where the estimation window increases by one observation at each time when computing new forecasts.

The hyperparameter $\lambda$ is set such that the in-sample fit, calculated in the initial estimation period, of the larger models (model MEDIUM and richer specification) is close to the in-sample fit of model SMALL, which is estimated without imposing any shrinkage. For example, when I set $\lambda$ parameter for the model MEDIUM, I minimize the difference:

$$
\left|R_{\text {pseudo_SMALL }}^{2}-R_{\text {pseudo_MEDIUM }}^{2}\right| \text {. }
$$

I repeat this procedure for all the models including many predictors. The in-sample fit 
is evaluated by the pseudo- $R^{2}$ (see Estrella (1998)) defined as:

$$
R_{\text {pseudo }}^{2}=1-\left(\frac{\ln l}{\ln c}^{\left(2 / T_{\text {in }}\right) \ln c}\right)
$$

where $\ln l=\sum_{t=1}^{T_{i n}}\left(y_{t} \times \ln \left(\widehat{P}_{t}^{i n}\right)+\left(1-y_{t}\right) \times \ln \left(1-\widehat{P}_{t}^{i n}\right), \ln c=T_{i n}(\bar{y} \times \ln (\bar{y})+(1-\bar{y}) \times \ln (1-\bar{y}))\right.$. In these expressions, $\bar{y}$ is the sample average of recession periods, $\widehat{P}_{t}^{i n}$ is the fitted (in-sample) recession probability obtained from (2.4) and (2.5) and $T_{i n}$ is the number of in-sample observations. Notice that $\ln c$ corresponds to the value of the log-likelihood function obtained by a model which includes just a constant term. $R_{\text {pseudo }}^{2}$ takes a value between 0 and 1 , and it has a similar interpretation to the usual $R^{2}$ obtained in linear models for real-valued variables. The value of $R_{\text {pseudo }}^{2}$ obtained from model SMALL in the in-sample period with $\lambda=1000$ (which implies no shrinkage) is around 0.70 .

Table 1 shows the values of $\lambda$ selected for our models. I consider both the case where the same shrinkage is imposed on all lags (i.e. matrix $\mathrm{I}_{K}$ ) and the one where we impose tighter shrinkage on predictors further in the past (using matrix $\mathrm{J}_{K}$ ).

\begin{tabular}{cccccc} 
Shrinkage & $\mathrm{R}_{\text {pseudio }}^{2}$ & $\lambda_{\text {Medium }}$ & $\lambda_{\text {LARGe }}$ & $\lambda_{\text {Vlarge }}$ & $\lambda_{\text {Giant }}$ \\
\hline $\mathrm{I}_{K}$ & 0.70 & 0.0244 & 0.0061 & 0.0042 & 0.001 \\
$\mathrm{~J}_{K}$ & 0.70 & 0.0732 & 0.018 & 0.008 & 0.002 \\
\hline \hline
\end{tabular}

Table 1: The values of $\lambda$ selected for different models given $R_{\text {pseudo }}^{2}=0.70$ for model SMALL.

In Table $1, \lambda$ tends to decrease as I add more variables, indicating that the model needs more shrinkage to prevent overfitting. Moreover, when I impose a tighter shrinkage on longer lags of the predictors, i.e. I use matrix $\mathrm{J}_{K}$, the optimal values of $\lambda$ are larger than when matrix $\mathrm{I}_{K}$ is used. This result is in line with the basic intuition and with previous studies (see De Mol et al. (2008)).

Out-of-sample forecasting results are evaluated using the Quadratic Probability Score, which is the counterpart of the mean squared forecast error in the models for real-valued variables (see, e.g., Christiansen et al. (2014)). It is defined as

$$
Q P S=\frac{2}{\left(T-T_{i n+1}\right)} \sum_{t=T_{i n}+1}^{T}\left(\widehat{P}_{t}-y_{t}\right)^{2},
$$

where $\widehat{P}_{t}$ indicates the posterior mean of the h-months-ahead forecasts calculated following (2.9) and (2.11). The value of the QPS is between 0 and 2 so that lower values indicate more accurate forecasts. 


\section{$4.2 \quad$ In-sample Results}

I first consider the in-sample fit of various models using the full sample period. In particular, I shrink the parameters of larger models to prevent overfitting, following the procedure described above in Section 4.1. The choice of $\lambda$ is based on the data included in the first in-sample period. Below, in Figure 1, I depict the plots of the fitted values for models SMALL, LARGE and GIANT. The reason to focus on these three models is that they represent different degrees of data availability. Model SMALL does not have any shrinkage and includes only the term spread and the federal funds rate, while model LARGE includes also stock market information and finally model GIANT includes the full information set available.

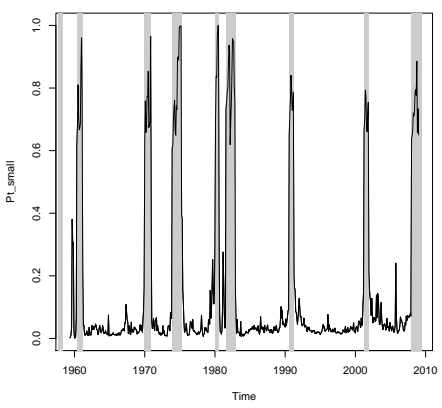

(a) Model SMALL

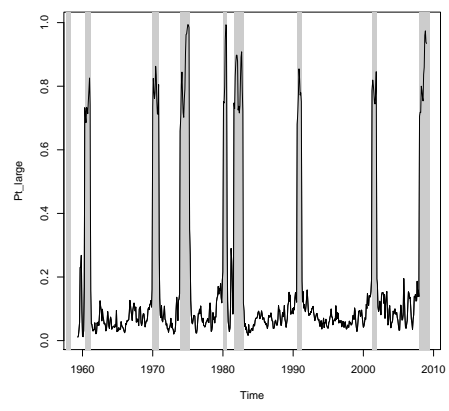

(b) Model LARGE

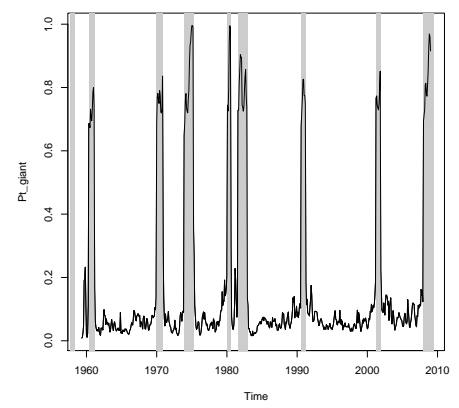

(c) Model GIANT

Figure 1: The in-sample fit with shrinkage.

As expected, when I impose shrinkage on the parameters, the in-sample fit of different model does not seem to differ substantially. This insight can be confirmed by looking at the values of the $R_{\text {pseudo }}^{2}$ for our models in Table 2 .

\begin{tabular}{cccccccc} 
Shrinkage on Lags & \multicolumn{1}{c}{ SP } & \multicolumn{1}{c}{ SMALL } & MEDIUM & LARGE & VLARGE & GIANT FACTORS \\
\hline $\mathrm{I}_{K}$ & 0.64 & 0.74 & 0.72 & 0.68 & 0.72 & 0.70 & 0.77 \\
$\mathrm{~J}_{K}$ & 0.64 & 0.74 & 0.69 & 0.75 & 0.68 & 0.67 & 0.77 \\
\hline \hline
\end{tabular}

Table 2: The values $R_{\text {pseudo }}^{2}$ for given $\lambda$ (see Table 1 ).

Remember that $\lambda$ is selected in such a way to achieve equal in-sample fit of model SMALL in the first estimation period (corresponding roughly to the half of the total observations available in the data). It is therefore normal that the final $R_{\text {pseudo }}^{2}$ values are different when I consider the entire time span of the analysis. However, the shrinkage seems to be working for both the case where $\mathrm{I}$ have $\mathrm{I}_{K}$ and $\mathrm{J}_{K}$. Adding more variables to the model, when shrinking, does not improve its fit. Notice, that I do not shrink the 
parameters of model FACTORS and thus it seems to have the best in-sample fit.

It is interesting to see how the models would perform in terms of in-sample predictions if I do not impose any shrinkage (i.e. set $\lambda$ large). In Figure 2, I depict the results when all the models are estimated without applying any shrinkage on the parameters.

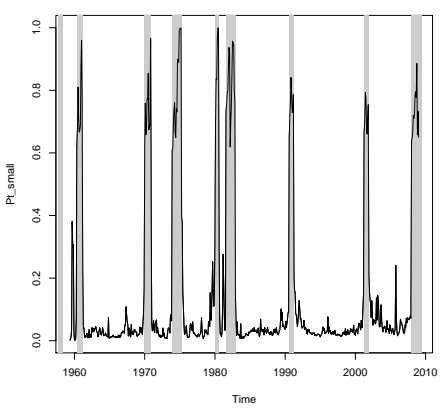

(a) Model SMALL

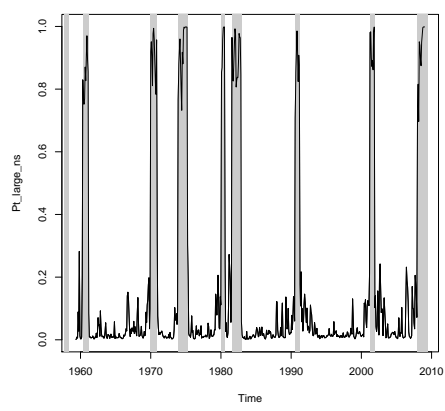

(b) Model LARGE

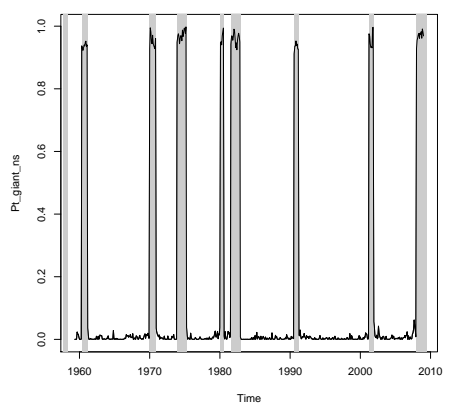

(c) Model GIANT

Figure 2: In-sample fit without shrinkage.

The figures already indicate that in the absence of shrinkage, as expected, larger models achieve very accurate in-sample fit. For example, in model GIANT the fitted values mimic the recession indicator almost perfectly. This good in-sample performance can also be seen in the $R_{\text {pseudo-values reported in Table } 3 .}^{2}$

\begin{tabular}{ccccccc}
\multicolumn{3}{c}{ SP } & SMALL & MEDIUM & \multicolumn{3}{c}{ LARGE } & VLARGE & GIANT FACTORS \\
\hline 0.64 & 0.74 & 0.75 & 0.82 & 0.89 & 0.95 & 0.77 \\
\hline
\end{tabular}

Table 3: The values of the $R_{\text {pseudo }}^{2}$ for the different models, with $\lambda=100$.

As expected, imposing no shrinkage on the parameters leads to very good in-sample fit, and it is monotonically increasing with the size of the model as smaller models are just subsets of the largest model. However, we have to bear in mind that good in-sample fit does not necessarily imply accurate forecasting performance out of sample. Actually, due to overfitting, it is likely that models with very high predictive accuracy in-sample may have very poor forecasting performance. Out-of-sample forecasts are examined in more detail in the next section.

\subsection{Out-of-sample Results}

I now turn to the out-of-sample forecasting performance of the models by looking at the estimated posterior mean probabilities of recession computed using (2.9) and (2.11). In Figure 3, I present the plots of the mean of the posterior predictive distributions, our 
point estimates for the probability of recession, one-month-ahead $(\mathrm{h}=1)$ using shrinkage method described above.

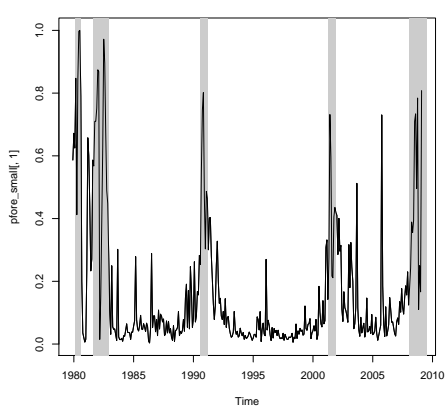

(a) Model SMALL

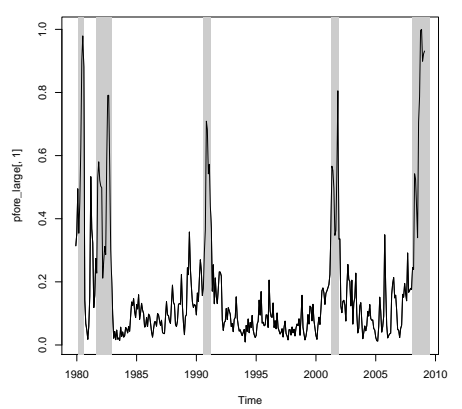

(b) Model LARGE

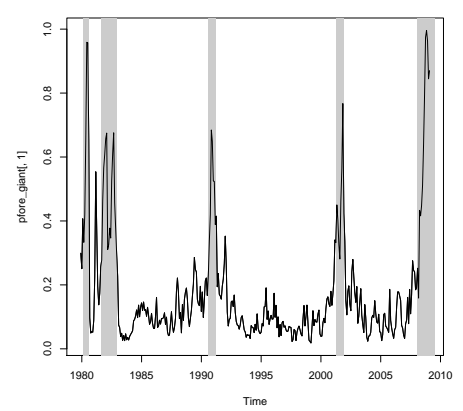

(c) Model GIANT

Figure 3: One-month-ahead forecasts with shrinkage.

The plots in Figure 3 already indicate that the shrinkage strategy works well in forecasting recessions in the near future. Model GIANT, which has more than 100 predictors, seems to provide pretty accurate one-month-ahead forecasts without producing any false alarm. An example of a false alarm is visible in the model SMALL around the year 2006, where the probability of recession in the next month reaches 0.7 , but as we can see that there was no recession around that time.

While interesting from a methodological perspective, and in a possible nowcasting setting, forecasting recessions one-month-ahead have not been the main focus of the literature. Studies as Chauvet and Potter (2005) and Nyberg (2010), among others, have focused on long-horizon recession forecasting, most commonly one-year-ahead. Therefore, in Figure 4 I report the plots for the 12-month-ahead forecasts, applying shrinkage to the parameters.

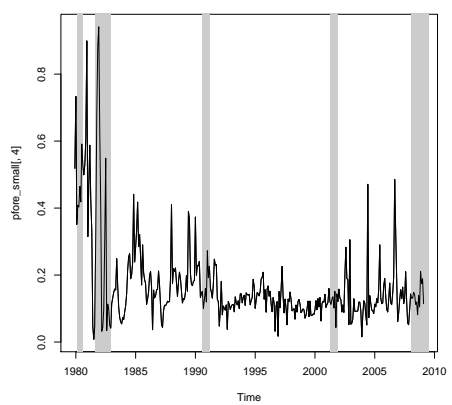

(a) Model SMALL

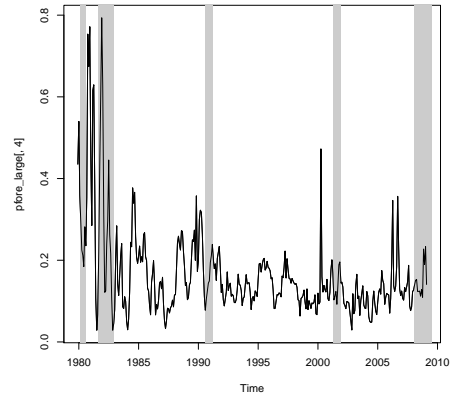

(b) Model LARGE

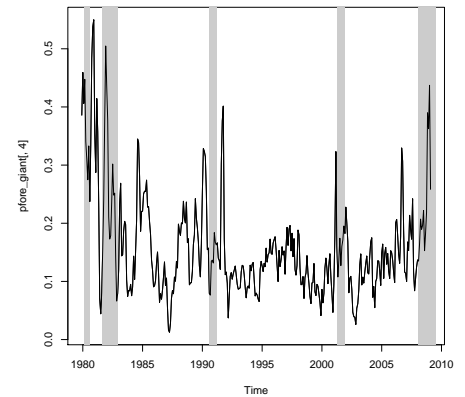

(c) Model GIANT

Figure 4: 12-month-ahead forecasts with shrinkage.

There are few things we need to be aware of, when examining these plots. It 
seems that larger models provide much less volatile forecasts compared with the model including only five variables (SMALL) and no shrinkage. While one-year-ahead recession forecasts in model GIANT never reach very high recession probabilities, it is pretty clear when the recession probability spikes with respect to non-recession periods. This result is line with the findings of Kauppi and Saikkonen (2008), where dynamic models seem to give weaker, albeit sharper signals of actual recessions in contrast of slowdowns of the economy during expansions. Moreover, it seems that model GIANT is able to forecast the recessions, without creating too many false alarms. Actually, most of the false alarms produced by the model GIANT are in proximity of the recession periods so they provide important information regarding the future state of the economy. Only around the year 1983, the model GIANT create a false alarm which is far from the subsequent recession. On the other hand, more parsimonious models such as SMALL and LARGE have difficulties in forecasting the early 1990s and 2000s recessions, together with the latest (2008-2009) recession. However, these two models do a good job in forecasting the recessions in the 80 's.

Figure 3 and 4 are useful to get a grasp of the forecasting performance of our models but numerical indicators are easier to interpret in comparing the predictive accuracy of the models under examination. Below, in Table 4, I report the QPS-statistics (4.1), for the models described in Section 3 for forecast horizons $\mathrm{h}=1,6,9$ and 12 , where $\lambda$ is set according to Table 1. I include the results both for the shrinkage independent of the lag order (i.e. we use the matrix $\mathrm{I}_{K}$ ) and with smaller $\lambda$ imposed on the higher order lags (matrix $\mathrm{J}_{K}$ ).

\begin{tabular}{cccccccccc} 
Shrinkage & \multicolumn{3}{c}{$\mathrm{I}_{K}$} \\
\hline & $\mathrm{h}=1$ & $\mathrm{~h}=6$ & $\mathrm{~h}=9$ & $\mathrm{~h}=12$ & $\mathrm{~h}=1$ & $\mathrm{~h}=6$ & $\mathrm{~h}=9$ & $\mathrm{~h}=12$ \\
\hline $\mathrm{SP}$ & 0.28 & 0.29 & 0.27 & 0.27 & 0.28 & 0.29 & 0.27 & 0.27 \\
SMALL & 0.13 & 0.25 & 0.27 & 0.27 & 0.13 & 0.25 & 0.27 & 0.27 \\
MEDIUM & 0.14 & 0.27 & 0.28 & 0.26 & 0.14 & 0.28 & 0.31 & 0.25 \\
LARGE & 0.11 & 0.23 & 0.28 & 0.27 & 0.13 & 0.22 & 0.24 & 0.25 \\
VLARGE & 0.11 & 0.22 & 0.24 & 0.24 & 0.14 & 0.21 & 0.23 & 0.23 \\
GIANT & 0.11 & 0.21 & 0.23 & 0.23 & 0.13 & 0.21 & 0.23 & 0.23 \\
FACTORS & 0.10 & 0.23 & 0.25 & 0.26 & 0.10 & 0.23 & 0.25 & 0.26 \\
\hline \hline
\end{tabular}

Table 4: Out-of-sample QPS statistics for the models with shrinkage and matrix $\mathrm{I}_{K}$ and $\mathrm{J}_{K}$.

It is clear that adding real activity predictors (going from SP to SMALL) improves considerably short-term forecasts while it does not seem to have a large effect on the 
longer term horizons. This result is likely to reflect the presence of information (as discussed in Section 3) about the coincident state of the economy at the time the forecast is computed. Even though the determination of recession periods reflect a somewhat subjective judgment, coincident indicators such as the ones included in SMALL and the larger dataset are strongly correlated with the NBER's definition of a recession. However, the long-term forecasts are largely unaffected by the inclusion of real economic activity indicators. This is due to the fact that the term-spread (already present in the simplest model) is a dominant leading indicator for recession periods. Nevertheless, increasing the set of explanatory variables, while shrinking the parameters toward zero, provides superior forecasting performance at all the forecast horizons. Model GIANT creates the most accurate forecasts between the specifications considered here. For example, 12-month-ahead forecasts obtained with model SP present $14 \%$ larger value of the out-of-sample QPS than model GIANT. However, the model FACTORS provides the best one-month-ahead forecasts.

As we can see in Table 4, imposing matrix $\mathrm{J}_{K}$, instead of the identity matrix, to shrink the parameters of larger models does not seem to influence a lot the out-of-sample performance. Only model LARGE seems to benefit from the additional shrinkage imposed on the predictors further in the past.

Finally, it is interesting to see how the models would perform in the case of noshrinkage. First, in Figures 5 and 6 , I provide plots of the posterior mean predictive distributions for the forecast horizons $h=1$ and $h=12$ with no shrinkage.

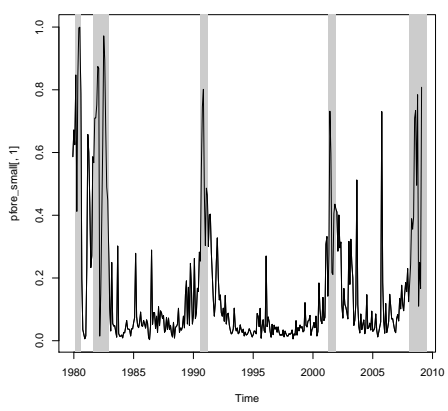

(a) Model SMALL

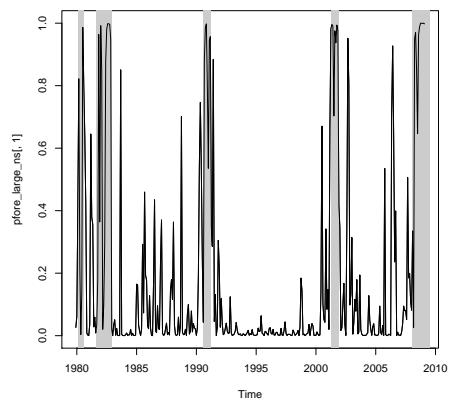

(b) Model LARGE

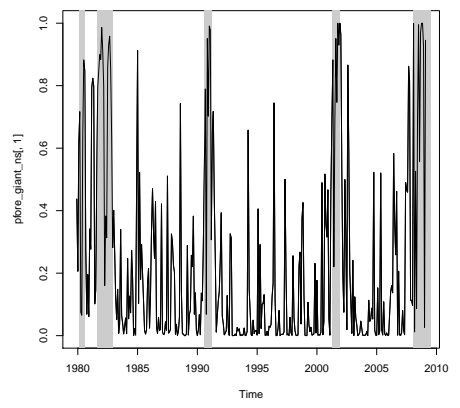

(c) Model GIANT

Figure 5: 1-month-ahead forecasts with no shrinkage. 


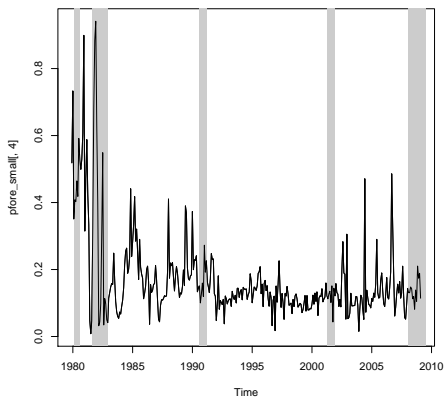

(a) Model SMALL

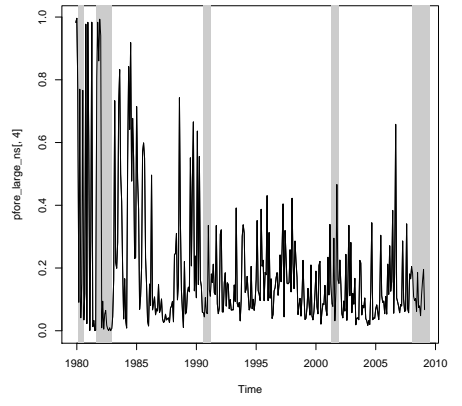

(b) Model LARGE

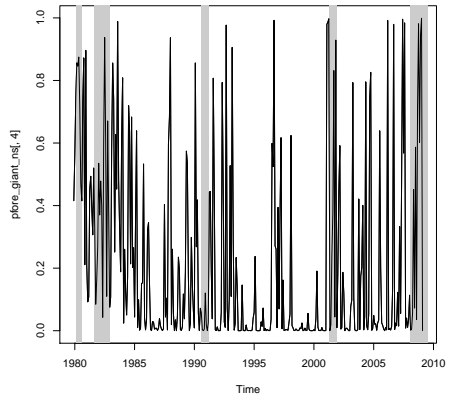

(c) Model GIANT

Figure 6: 12-month-ahead forecasts with no shrinkage.

As expected, the forecasting performance seems to deteriorate greatly when I do not impose any shrinkage on larger models. The forecasts become extremely volatile at both long and short-term horizons, creating many false alarms and giving no useful information to policy makers. To confirm these findings, in Table 5, I present the QPS statistics for the forecasting models estimated by setting $\lambda$ to very large values (i.e. no shrinkage imposed).

\begin{tabular}{ccccc} 
Model & $\mathrm{h}=1$ & $\mathrm{~h}=6$ & $\mathrm{~h}=9$ & $\mathrm{~h}=12$ \\
\hline SP & 0.23 & 0.26 & 0.26 & 0.26 \\
SMALL & 0.13 & 0.23 & 0.27 & 0.25 \\
MEDIUM & 0.20 & 0.30 & 0.32 & 0.31 \\
LARGE & 0.13 & 0.32 & 0.31 & 0.33 \\
VLARGE & 0.16 & 0.34 & 0.36 & 0.35 \\
GIANT & 0.17 & 0.35 & 0.32 & 0.33 \\
FACTORS & 0.10 & 0.23 & 0.25 & 0.26 \\
\hline \hline
\end{tabular}

Table 5: QPS for the models with no shrinkage.

We see that imposing a flat prior deteriorates the forecasting performance of larger models substantially. This confirms the need of shrinkage when increasing the set of explanatory variables. This is expected as the models with a large number of predictors suffer from overfitting.

Looking at the empirical results gathered in this section, it seems that model GIANT generally provides the best out-of-sample performance, at least for forecast horizons longer than one month. Moreover, as we saw in Figure 4, model GIANT provides useful insights to predict the state of the economy when going beyond the actual QPS values. While the computed recession probabilities never reach high values, the spikes during the economic downturns are clearly visible. The good performance of large models is a 
remarkable result also in the light of actual implementability. Nowadays, large datasets are available to central banks, statistical offices and many other institutions, so being able to use all the information available to forecast the future state of the economy is highly beneficial. Bayesian shrinkage examined in this paper allows us to deal with large information set without incurring into the problem of overfitting and, as we have seen above, giving competitive out-of-sample forecasts.

\section{Conclusions}

The use of large datasets in macroeconomic forecasting has been widely adopted in the last few decades. However, in forecasting business cycle recession periods, the literature has focused on the use of a small number of predictive variables. A few attempts to incorporate large information sets into the analysis have relied on the use of factor-based models (see, e.g., Christiansen et al. (2014)), where the extracted factors are employed in the probit model. In this study, I adopt a Bayesian shrinkage approach to estimate probit models which include a large number of predictive variables. I set the shrinkage proportionally to the number of predictors included so that the (in-sample) predictive power of larger models is equal to the specification with only a handful of predictors. In terms of the in-sample fit, the methodology works well, preventing overfitting issues even for the models with more than 100 predictors. The ability of using a large number of predictors, without estimating latent factors is the key contribution of this research. Bayesian shrinkage facilitates economic interpretation of the predictors in the analysis (contrary to factor-model based forecasts, which rely on extracted common components with no clear economic interpretation).

I find that the probit model including all the predictive variables yields the best out-of-sample predictions for all forecast horizons. Models including a large number of predictors are able to beat the factor-based model, albeit the latter gives us the best one-month-ahead forecasts. Moreover, the forecasts from the largest model, even for the 12-month horizon, do not present evident false alarms, while they provide a clear indication of when future recession is likely. This result holds true for all the recession periods we have in our sample.

The models we have considered here are static, i.e. they do not include any dynamics of the recession indicator or the latent variable underlying it. While the presence of large information sets, especially the inclusion of coincident indicators such as industrial 
production, should already compensate for missing dynamics, it could be interesting to examine in the future research (outside the scope of this paper) dynamic models similar to Chauvet and Potter (2005). Another interesting extension of this paper lies in the priors' selection. In this study, I shrink all the parameters toward 0. However, we know from previous literature that a subset of predictors are especially useful in recession forecasting. It could be beneficial to impose priors that reflect this knowledge, i.e. shrinking toward non-zero values, possibly drawn from previous studies such as Nyberg (2010) and Wright (2006). Finally, the ability to include large amount of variables is desirable in a real-time environment, where the decision makers might have access to large data, but do not have a clear guidance on which variables to select. Examining the forecasting performance of our models in a real-time analysis, where we take into account the time delays due to the publication lags of different variables including the binary recession indicator, can also be the subject of future research.

\section{References}

James H. Albert and Siddhartha Chib. Bayesian analysis of binary and polychotomous response data. Journal of the American Statistical Association, 88(422):pp. 669-679, 1993.

Jushuan Bai and Serena Ng. Determining the Number of Factors in Approximate Factor Models. Econometrica, 70(1):191-221, 2002.

Marta Banbura, Domenico Giannone, and Lucrezia Reichlin. Large Bayesian Vector Auto Regressions. Journal of Applied Econometrics, 25(1):71-92, 2010.

Marcelle Chauvet and Simon Potter. Forecasting recessions using the yield curve. Journal of Forecasting, 24(2):77-103, 2005.

Zhihong Chen, Azharand Iqbal, and Huiwen Lai. Forecasting the probability of US recessions: a Probit and dynamic factor modelling approach. Canadian Journal of Economics, 44(2):651-672, 2011.

Charlotte Christiansen, Jonas N. Eriksen, and Stig V. Møller. Forecasting US recessions: The role of sentiment. Journal of Banking and Finance, 49(C):459-468, 2014.

Christine De Mol, Domenico Giannone, and Lucrezia Reichlin. Forecasting using a 
large number of predictors: Is Bayesian shrinkage a valid alternative to principal components? Journal of Econometrics, 146(2):318-328, 2008.

Michael Dueker. Strengthening the case for the yield curve as a predictor of U.S. recessions. Federal Reserve Bank of St. Louis Review, 79:41-51, 1997.

Arturo Estrella. A New Measure of Fit for Equations with Dichotomous Dependent Variables. Journal of Business and Economic Statistics, 16(2):198-205, 1998.

Arturo Estrella and Frederic S. Mishkin. Predicting U.S. Recessions: Financial Variables As Leading Indicators. Review of Economics and Statistics, 80(1):45-61, 1998.

Sebastian Fossati. Forecasting U.S. Recessions with Macro Factors. Working Papers 2013-3, University of Alberta, Department of Economics, 2013.

Heikki Kauppi and Pentti Saikkonen. Predicting U.S. Recessions with Dynamic Binary Response Models. Review of Economics and Statistics, 90(4):777-791, 2008.

Massimiliano Marcellino, James H. Stock, and Mark W. Watson. A comparison of direct and iterated multistep AR methods for forecasting macroeconomic time series. Journal of Econometrics, 135(1-2):499-526, 2006.

Henri Nyberg. Dynamic probit models and financial variables in recession forecasting. Journal of Forecasting, 29(1-2):215-230, 2010.

Henri Nyberg. A bivariate autoregressive probit model: Business cycle linkages and transmission of recession probabilities. Macroeconomic Dynamics, 18:838-862, 2014.

Richard Startz. Binomial Autoregressive Moving Average Models With an Application to U.S. Recessions. Journal of Business and Economic Statistics, 26:1-8, 2008.

James H. Stock and Mark W. Watson. Macroeconomic forecasting using diffusion indexes. Journal of Business and Economic Statistics, 20(2):147-62, 2002.

James H. Stock and Mark W. Watson. Generalized Shrinkage Methods for Forecasting Using Many Predictors. Journal of Business and Economic Statistics, 30(4):481-493, 2012.

Jonathan H. Wright. The yield curve and predicting recessions. Finance and Economics Discussion Series 2006-07, Board of Governors of the Federal Reserve System (U.S.), 2006. 
A. Zellner. An Introduction to Bayesian Inference in Econometrics. Wiley Classics Library. Wiley, 1971. 